\title{
Comments on interactions in the SUSY models
}

\author{
Sudhaker Upadhyay ${ }^{1, \mathrm{a}}$, Alexander Reshetnyak ${ }^{2, \mathrm{~b}}$, Bhabani Prasad Mandal ${ }^{1, \mathrm{c}}$ \\ ${ }^{1}$ Department of Physics, Banaras Hindu University, Varanasi 221005, India \\ 2 Institute of Strength Physics and Materials Science of SB RAS, Tomsk 634021, Russia
}

Received: 2 November 2015 / Accepted: 16 June 2016 / Published online: 12 July 2016

(c) The Author(s) 2016. This article is published with open access at Springerlink.com

\begin{abstract}
We consider special supersymmetry (SUSY) transformations with $m$ generators $\overleftarrow{s}_{\alpha}$, for some class of models and study the physical consequences when making the Grassmann-odd transformations to form an Abelian supergroup with finite parameters and a set of group-like elements with finite parameters being functionals of the field variables. The SUSY-invariant path integral measure within conventional quantization scheme leads to the appearance of the Jacobian under a change of variables generated by such SUSY transformations, which is explicitly calculated. The Jacobian implies, first of all, the appearance of trivial interactions in the transformed action, and, second, the presence of a modified Ward identity which reduces to the standard Ward identities in the case of constant parameters. We examine the case of the $N=1$ and $N=2$ supersymmetric harmonic oscillators to illustrate the general concept by a simple free model with $(1,1)$ physical degrees of freedom. It is shown that the interaction terms $U_{\text {tr }}$ have a corresponding SUSYexact form: $U_{\text {tr }}=\left(V_{(1)} \overleftarrow{s} ; V_{(2)} \overleftarrow{s} \overleftarrow{s}\right)$ generated naturally under such generalized formulation. We argue that the case of a non-trivial interaction cannot be obtained in such a way.
\end{abstract}

\section{Introduction}

Supersymmetric theories are invariant under SUSY transformations which relate the bosonic and fermionic degrees of freedom present in the theories and were proposed initially with the motivation of studying the fundamental interactions in a unified manner. The generators of SUSY transformations satisfy the Lie superalgebra relations, which are closed under a combination of the commutators and anticommutators. The local or non-linear versions of Lie superalgebra constructions have been extended to various field-theoretic

\footnotetext{
a e-mail: sudhakerupadhyay@gmail.com

be-mail: reshet@ispms.tsc.ru

c e-mail: bhabani.mandal@gmail.com
}

models such as superstring theories [1], supergravity [2,3] (for modern developments see Refs. [4,5]) and higher-spin field theories [6-11]. SUSY theory provides a bosonic superpartner to each fermion presented in the theory and vice versa. This indicates whether $N=1$ (with one fermionic generator in terms of Dirac spinor) SUSY has to be a perfect symmetry of nature; then each set of superpartners must have the same set of quantum numbers with as the only difference the spin. Despite the beauty of all these unified theories, the SUSY theory has not been supported by experimental evidence so far, but it remains one of the problems of the LHC experimental program.

Some variants of SUSY have also become interesting topics in quantum mechanics [12] due to the link to exactly solvable models. SUSY and its breaking have been studied in various simple quantum mechanical systems involving a spin$1 / 2$ particle moving in one direction [13,14]. The supersymmetric Hamiltonian may be presented in terms of the supercharges which generate the SUSY transformations. A path integral formulation of SUSY in quantum mechanics was first analyzed by Salomonson and van Holten [15]. Further, by using SUSY methods, the tunneling rate through quantum mechanical barriers was accurately determined [16-19].

The SUSY transformations, when applied for the gauge theory together with special global SUSY transformations, known as BRST transformations [20-22], have also been explored in a more effective way [23,24]. The BRST symmetry and the associated concept of BRST cohomology provide the commonly used quantization methods in Lagrangian $[25,26]$ and Hamiltonian $[27,28]$ formalisms for the gauge and string theories $[29,30]$. The BRST symmetry was generalized [26] to the case of an infinitesimal field-dependent (FD) transformation parameter $\mu, \mu^{2}=0$, within the fieldantifield formalism $[25,26]$ in [26], in order to prove the independence from small gauge variations of the path integral for arbitrary gauge theory. A further generalization [31] was made in Yang-Mills theories with $R_{\xi}$-gauges by making the transformation parameter finite and field-dependent, as one 
considers a sequence of infinitesimal field-dependent BRST transformations (for a numeric parameter $\kappa$ ) with some applications [32-57]. Another way to consider a finite fielddependent parameter in Yang-Mills theory was inspired by the research devoted to so-called soft BRST symmetry breaking problem [58], with reference to the Gribov problem [59], which also involves the Zwanziger proposal [60] for a horizon functional joined additively to a BRST-invariant quantum action. In fact, the horizon functional in $R_{\xi}$-gauges with small $\xi$ was found explicitly in [58] [see Eq. (5.20) therein], by using field-dependent BRST transformations with a small odd-valued parameter, which then was extended to a finite case [61]. The case of finite field-dependent BRST transformations for general gauge theories was considered in [62], and for the case of BRST-antiBRST symmetry [63-65] in [66-70], with the original algorithm of the calculation of the functional Jacobians (for a comparative analysis of BRST symmetry, see [71]).

At the same time, analogous properties of space-time SUSY transformations (with Grassman-odd parameters) have never been found. Therefore, in spite of the fact that BRST transformations are realized in an extended field space with initial classical and ghost, antighost, Nakanishi-Lautrup fields, and are reminiscent of the gauge transformations, a similar application of the SUSY transformations in the path integral with FD Grassman-odd parameters to fieldtheoretical models (without auxiliary field variables to be introduced via the Faddeev-Popov prescription [72]) provides us with an opportunity to apply the above research to study the influence of SUSY transformations on the quantum action structure.

In this paper, we consider a generalization of SUSY transformations to the case of an $m$-parametric Lie superalgebra with the transformation parameters being finite and fielddependent. In this way, the resulting transformations remain a symmetry of the supersymmetric action. Under generalized SUSY transformations with arbitrary field-dependent parameters the functional measure, however, is not invariant. This leads to a non-trivial Jacobian for the functional measure and therefore to a modification of the quantum action by non-quadratic terms being a SUSY-exact contribution. For some choices of parameters, the generalized SUSY transformations amount to a precise change in the exponent action. We illustrate these results by an example of a free toy model with $(1,1)$ physical degrees of freedom, describing a supersymmetric harmonic oscillator with the generalized $N=1$ and $N=2$ SUSY transformations. In such a theory, the interaction terms emerge naturally within the functional integral under the generalized SUSY with specific parameters.

The paper is organized as follows. In Sect. 2, we study the generalized SUSY transformations with $m$ Grassman-odd parameters for a general supersymmetric invariant theory, calculate the corresponding Jacobian of the change of vari- ables, derive the standard and modify Ward identities, and classify the interactions. In Sect. 3, we illustrate the example of generalized SUSY transformations in a supersymmetric harmonic oscillator with $(1,1)$ degrees of freedom, in such a way that the trivial interaction terms are produced by generalized $N=1, N=2$ SUSY transformations from the functional measure. Finally, we summarize the results in the Conclusions.

We use the DeWitt condensed notation and the conventions of $[31,62,66-69]$, e.g., $\varepsilon(F)$ denotes the value of the Grassmann parity of a quantity $F$.

\section{Generalized SUSY transformations}

Here, we investigate a finite field-dependent SUSY (FSUSY) transformation for general supersymmetric invariant theories (following the technique developed both in [31] and in [62, 66-69]). To this end, we first define the SUSY transformation with infinitesimal Grassmann-odd constant parameters $\epsilon^{\alpha}$, $\alpha=1, \ldots, m, \varepsilon\left(\epsilon^{\alpha}\right)=1$, leaving invariant an action $S(q)$ of generic variables $q^{i}, i=1, \ldots, n, n=\left(n_{+}, n_{-}\right), \varepsilon\left(q^{i}\right)=$ $\varepsilon_{i}$ :

$$
\begin{aligned}
\delta_{\epsilon} q^{i} & =\mathcal{R}_{\alpha}^{i}(q) \epsilon^{\alpha}=q^{i} \overleftarrow{s}_{\alpha} \epsilon^{\alpha}: \quad S\left(q+\delta_{\epsilon} q\right)=S(q)+o(\epsilon) \\
& \Longleftrightarrow S(q) \overleftarrow{\partial}_{i} \mathcal{R}_{\alpha}^{i}(q)=S(q) \overleftarrow{s}_{\alpha}=0,
\end{aligned}
$$

where $\overleftarrow{\partial}_{i} \equiv \frac{\overleftarrow{\delta}}{\delta q^{i}}$ and $\mathcal{R}_{\alpha}^{i}(q), \overleftarrow{s}_{\alpha}, \varepsilon\left(\mathcal{R}_{\alpha}^{i}, \overleftarrow{s}_{\alpha}\right)=\left(\varepsilon_{i}+1,1\right)$ are, respectively, the generators of SUSY transformation of generic variables $q^{i}$ and those acting on functionals $F(q)$. We suppose that the generators of SUSY transformations satisfy the Abelian anticommutator relations:

$\left[\delta_{\epsilon_{1}}, \delta_{\epsilon_{2}}\right]=0 \Longleftrightarrow\left\{\overleftarrow{s}_{\alpha}, \overleftarrow{s}_{\beta}\right\}=0$

The group transformations with finite parameters, $\epsilon^{\alpha}, q^{i} \rightarrow$ $q^{\prime i}=q^{i}(q \mid \epsilon)$, may be restored by two equivalent ways from the Lie equations and the requirement for any $\overleftarrow{s}_{\alpha}$-closed functional $F(q)$ to be invariant with respect to right group transformations:

$$
\begin{gathered}
q^{i}(q \mid \epsilon) \overleftarrow{\partial}_{\alpha}=q^{i}(q \mid \epsilon) \overleftarrow{s}_{\alpha}\left(\text { for } \overleftarrow{\partial}_{\alpha} \equiv \frac{\overleftarrow{\partial}}{\partial \epsilon^{\alpha}}\right) \\
\Longleftrightarrow F\left(q^{i}(q \mid \epsilon)\right)=F(q)
\end{gathered}
$$

For a $t$-rescaled argument $\epsilon^{\alpha} \rightarrow t \epsilon^{\alpha}$ of $q^{i}(q \mid t \epsilon)$, the form of Lie equations is equivalent to (3), with a formal solution for constant $\epsilon^{\alpha}$,

$\frac{d}{\mathrm{~d} t} q^{i}(q \mid t \epsilon)=q^{i}(q \mid t \epsilon) \overleftarrow{s}_{\alpha} \epsilon^{\alpha} \Longrightarrow q^{i}(q ; t \epsilon)=q^{i} \exp \left\{t \overleftarrow{s}_{\alpha} \epsilon^{\alpha}\right\}$ 
so that the set of finite transformations form an Abelian group $G=\left\{g(\epsilon): g(\epsilon)=\exp \left\{t \overleftarrow{s}_{\alpha} \epsilon^{\alpha}\right\}, \quad g\left(\epsilon_{1}\right) g\left(\epsilon_{2}\right)=\right.$ $\left.g\left(\epsilon_{2}\right) g\left(\epsilon_{1}\right)\right\}$, for constant $\epsilon^{\alpha}$. For field-dependent $\epsilon^{\alpha}=$ $\epsilon^{\alpha}(q)$ having no explicit dependence on space-time coordinates $x^{\mu}, \partial_{\mu} \epsilon^{\alpha}(q)=0$, the set of algebraic elements $\mathcal{G}=\left\{\tilde{g}_{\text {lin }}(\epsilon(q)):=1+\overleftarrow{s}_{\alpha} \epsilon^{\alpha}(q)\right\}$ forms a non-linear algebra which corresponds to a set of formal group-like finite elements:

$\tilde{G}=\left\{\tilde{g}(\epsilon(q)): \tilde{g}=1+\sum_{i=1}^{m} \frac{1}{i !}\left[\prod_{k=1}^{i}\left(\overleftarrow{s}_{\alpha_{k}}\right) \prod_{k=1}^{i} \epsilon^{\alpha_{k+1-i}}(q)\right]\right\}$

obtained as the solution for $F\left(q^{i}(q \mid \epsilon(q))\right)=F(q)$ in (3) with finite FD $\epsilon^{\alpha}(q)$, as in [66-69]. Note that in the case $m=$ 1,2 we have the representations of finite BRST and BRSTantiBRST group (4) and group-like elements (5) [62, 66-69].

We refer to SUSY transformation generalized in such a way, an FSUSY transformation. Another way to derive FSUSY transformations for the $N=1$-parametric subset from $G$, i.e. for $m=1$, can be done by rendering the infinitesimal parameter $\epsilon^{1} \equiv \epsilon$ field dependent through a continuous interpolation of an arbitrary parameter $\kappa(0 \leq \kappa \leq 1)$, following Ref. [31]: $\left(q^{i}(\kappa=0) ; q^{i}(\kappa=1)\right)=\left(q^{i} ; q^{i}(q \mid \epsilon)\right)$.

An infinitesimal field-dependent SUSY transformation can be defined as

$\mathrm{d} q^{i}(\kappa)=\mathcal{R}^{i}(q) \epsilon^{\prime}(q(\kappa)) \mathrm{d} \kappa$,

where $\epsilon^{\prime}(q(\kappa)) \mathrm{d} \kappa$ is an infinitesimal field-dependent parameter. An FSUSY transformation with a finite field-dependent parameter can now be constructed by integrating such an infinitesimal transformation from $\kappa=0$ to $\kappa=1$, as follows:

$q^{i}(q \mid \epsilon) \equiv q^{i}+\mathcal{R}^{i}(q) \epsilon(q)$, where $\epsilon(q)=\int_{0}^{1} \mathrm{~d} \kappa \epsilon^{\prime}(q(q \mid \kappa))$.

Note that in the case of $m>1$ it is impossible to restore FSUSY transformations in this way explicitly using (6) by a simple integration over auxiliary $\kappa$ (for details see [31]).

Turning to FSUSY transformations with $m$ odd parameters, we see that such transformations remain a symmetry of the supersymmetric action, which we suppose to describe non-degenerate non-gauge theory, whereas the path integral measure will not be invariant under such a transformations and thereby will lead to a non-trivial Jacobian for a corresponding change of variables in the generating functional $Z(J)$ of Green's functions with external sources, $J_{i}$ $\left(\varepsilon\left(J_{i}\right)=\varepsilon_{i}\right)$ and in the path integral $Z(0)=Z_{0}$ :

$$
\begin{aligned}
& \begin{array}{l}
Z(J)=\int \mathcal{D} q \exp \left\{\frac{l}{\hbar}\left[S(q)+J_{i} q^{i}\right]\right\}, \\
Z_{0}=\int \mathcal{I}_{q} \text { with } \mathcal{I}_{q \tilde{g}(\epsilon)}=J(q) \mathcal{I}_{q}, \\
\text { where } J(q)=\operatorname{Sdet}\left\|q^{i}(q \mid \epsilon(q)) \overleftarrow{\partial}_{j}\right\| \\
=\exp \left\{\operatorname{Str} \ln \left(\delta_{j}^{i}+M_{j}^{i}(q, \epsilon)\right)\right\} \text { for } M_{j}^{i}(q, \epsilon) \\
=\Delta q^{i}(q \mid \epsilon) \overleftarrow{\partial}_{j},
\end{array}
\end{aligned}
$$

which vanishes when $\epsilon^{\alpha}=$ const, $\left.M_{j}^{i}(q, \epsilon)\right|_{\epsilon=\text { const }}=0$. The Jacobian can be calculated explicitly, following the receipt $[62,66-70]$ and also by using the Green's function method [73]. The latter approach, using $t$-rescaled parameters $t \epsilon^{\alpha}(4)$ and the inverse (formal) transformations $\tilde{g}^{-1}(\epsilon(q))$ :

$$
q^{i}(q \mid t \epsilon) \tilde{g}^{-1}(\epsilon(q))=q^{i} \Longrightarrow q^{i}\left(q^{\prime} \mid t \epsilon\right) \overleftarrow{\partial}_{\alpha}=-t q^{i} \overleftarrow{s}_{\alpha}
$$

assumes that the representation for $\ln J(q)$ given by (9) reads

$$
\begin{aligned}
\ln J(q) & =\operatorname{sTr} \ln \left(\delta_{j}^{i}-q^{i}\left(q^{\prime} \mid \epsilon\right) \overleftarrow{\partial}_{\alpha}\left(\epsilon^{\alpha} \overleftarrow{\partial}_{j}\right)\right) \Longrightarrow \frac{d}{\mathrm{~d} t} \ln J(q) \\
& =-\operatorname{tr}_{G}\left([e+t m]^{-1} m\right), \quad m_{\beta}^{\alpha}=\epsilon^{\alpha} \overleftarrow{s}_{\beta}
\end{aligned}
$$

where $(e)_{\beta}^{\alpha}$ and $\operatorname{tr}_{G}$ denote $\delta_{\beta}^{\alpha}$ and the trace over the indices of the matrix $G$. In deriving (11) we have used the fact that in differentiating with respect to $t$, the first of the above equalities reads

$$
\begin{aligned}
& G_{j}^{i}\left[q^{j} \overleftarrow{s}_{\alpha}\right]\left[\epsilon^{\alpha}(q) \overleftarrow{\partial}_{i}\right](-1)^{\varepsilon_{i}} \text { and follows from } \\
& G_{j}^{i}+t\left[q^{i} \overleftarrow{s}_{\alpha}\right]\left[\epsilon^{\alpha}(q) \overleftarrow{\partial}_{k}\right] G_{j}^{k}=\delta_{j}^{i}
\end{aligned}
$$

From the latter representation, we find

$\epsilon^{\alpha}(q) \overleftarrow{\partial}_{k} G_{j}^{k}=\left([e+t m]^{-1}\right)_{\beta}^{\alpha}\left(\epsilon^{\beta}(q) \overleftarrow{\partial}_{j}\right)$

so that after substitution in the first term of (12) we get the representation for the last quantity in (11), which after integrating leads to the final result for the Jacobian (because of $\ln J(q(0))=0)$,

$J(q(\epsilon))=\exp \left\{-\operatorname{tr}_{G} \ln ([e+m])\right\}$.

The Jacobian for $m=1,2$ is reduced to already known Jacobians for $N=1,2$ for finite FD BRST transformations with nilpotent $\overleftarrow{s}, \overleftarrow{s}_{a}, a=1,2$. For functionally-independent FD $\epsilon_{\alpha}(q)$ the Jacobian is not $\overleftarrow{s}_{\alpha}$-closed, in general, whereas for an $\overleftarrow{S}_{\alpha}$-potential (therefore functionally-dependent) we 
have the parameters

$\hat{\epsilon}^{\alpha}(q)=\frac{1}{(m-1) !} \Lambda(q) \varepsilon^{\alpha \alpha_{1} \ldots \alpha_{m-1}} \overleftarrow{s}_{\alpha_{1}} \ldots \overleftarrow{s}_{\alpha_{m-1}}$,

for $\varepsilon^{12 \ldots m}=1$ and $\varepsilon^{\alpha_{0} \alpha_{1} \ldots \alpha_{m-1}} \varepsilon_{\alpha_{m-1} \ldots \alpha_{1} \alpha_{0}}=m$ !

with potential arbitrary functional, $\Lambda(q), \varepsilon(\Lambda)=m$, and with completely antisymmetric tensors $\varepsilon^{\alpha_{0} \alpha_{1} \ldots \alpha_{m-1}}$, $\varepsilon_{\alpha_{m-1} \ldots \alpha_{1} \alpha_{0}}$ the Jacobian is $\overleftarrow{s}_{\alpha}$-closed.

Due to the equivalence theorem [74], the change of variables in $Z(J)$ and in the path integral $Z_{0}$ generated by FSUSY transformations (in terms of the integrand),

$$
\begin{aligned}
\mathcal{I}_{q \tilde{g}(\epsilon)} & =J(q) \mathcal{I}_{q}=D q \exp \left\{\frac{l}{\hbar}\left[S(q)+\imath \hbar \operatorname{tr}_{G} \ln ([e+m])\right]\right\} \\
& =D q \exp \left\{\frac{l}{\hbar}\left[S(q)+S_{1}(q, \epsilon(q))\right]\right\}
\end{aligned}
$$

leads to the same quantum theory, $Z_{0}=Z_{\epsilon}$, with the same conventional $S$-matrix. At the same time, a representation for the transformed action, $S(q, \epsilon(q))=S(q)+S_{1}(q, \epsilon(q))$, should be supersymmetrically invariant: $S(q, \epsilon(q)) \overleftarrow{S}_{\alpha}=0$. FSUSY transformations which satisfy the above must obey the condition

$S_{1}(q, \epsilon(q)) \overleftarrow{s}_{\alpha}=\imath \hbar \operatorname{tr}_{G} \ln ([e+m]) \overleftarrow{s}_{\alpha}=0$

In particular, an $N=m$ FSUSY transformations $\tilde{g}\left(\hat{\epsilon}^{\alpha}(q)\right)$ with FD parameters (15) for any potential $\Lambda(q)$ satisfy the condition (17).

Therefore, only trivial interactions, $U_{\mathrm{tr}}(q)$ can be generated (locally) by FSUSY transformations in the path integral, which are characterized by the condition $U_{\text {tr }}(q) \overleftarrow{s}_{\alpha}=0$ whereas the non-trivial interactions $U(q)$ which lead to a different $S$-matrix should satisfy the requirement

$U(q) \overleftarrow{s}_{\alpha}=0: \quad U(q) \neq V^{\alpha}(q) \overleftarrow{s}_{\alpha} \forall V^{\alpha}(q)$

For $m=1, m=2$ FSUSY transformations, the corresponding Jacobians (for functionally dependent $\epsilon_{a}=\Lambda \overleftarrow{s}_{a}$, $\left(\epsilon_{a}, \overleftarrow{s}^{a}\right)=\left(\varepsilon_{a b} \epsilon^{b}, \varepsilon^{a b} \overleftarrow{s}_{b}\right)$ with antisymmetric $\varepsilon_{a b}=-\varepsilon_{b a}$ and $\varepsilon^{a b}: \varepsilon^{a b} \varepsilon_{b c}=\delta_{c}^{a}$, under the normalization $\varepsilon^{12}=1$ )

$$
\begin{aligned}
J_{(1)}(q(\epsilon)) & =(1+\epsilon \overleftarrow{s})^{-1} \Rightarrow J_{(1)} \overleftarrow{s}=0 \text { and } J_{(2)}\left(q\left(\Lambda \overleftarrow{s}_{a}\right)\right) \\
& =\left(1+\frac{1}{2} \Lambda \overleftarrow{s}_{a} \overleftarrow{s}^{a}\right)^{-2} \Rightarrow J_{(2)} \overleftarrow{s}_{a}=0
\end{aligned}
$$

and lead only to trivial interactions. The invariance of the integrand $\mathcal{I}_{q}(8)$ with respect to FSUSY with constant parameters $\epsilon^{\alpha}$ leads to the presence of the Ward identities for $Z(J)$;

$$
\begin{aligned}
& J_{i}\left\langle q^{i} \overleftarrow{S}_{\alpha}\right\rangle_{J}=0 \text { where }\langle A(q)\rangle_{J}=Z^{-1}(J) \\
& \quad \times \int \mathcal{D} q A(q) \exp \left\{\frac{l}{\hbar}\left[S(q)+J_{i} q^{i}\right]\right\}, \quad\langle 1\rangle_{J}=1
\end{aligned}
$$

with a source-dependent average expectation value for a certain functional $A(q)$ corresponding to a given action $S(q)$. In turn, the property (16) with account taken of (14), for FD FSUSY transformations means the presence of a so-called modified Ward identity depending on the FD parameters $\epsilon(q)$ :

$$
\begin{aligned}
& \left\langle\exp \left\{\frac{l}{\hbar} J_{i} q^{i} \sum_{i=1}^{m} \frac{1}{i !}\left[\prod_{k=1}^{i}\left(\overleftarrow{s}_{\alpha_{k}}\right) \prod_{k=1}^{i} \epsilon^{\alpha_{k+1-i}}(q)\right]\right\}\right. \\
& \left.\quad \times \exp \left\{-\operatorname{tr}_{G} \ln ([e+m])\right\}\right\rangle_{J}=1
\end{aligned}
$$

(for $m_{\beta}^{\alpha}=\epsilon^{\alpha}(q) \overleftarrow{s}_{\beta}$ ), which reduces to (20) for constant $\epsilon^{\alpha}$.

In the case $m=1$ (but not for $m>1$ ) FSUSY may also be considered by evaluation of the Jacobian according to [31], restricted by infinitesimal FD parameter $\epsilon^{\prime}(q(\kappa))$ according to the definition of the change of variables $q^{i}(\kappa) \rightarrow q^{i}(\kappa+$ $d \kappa)$ with the Jacobian $J(\kappa)(9)$ :

$$
\begin{aligned}
& \mathcal{D} q(\kappa+d \kappa)=J(\kappa) \mathcal{D} q(\kappa) \stackrel{\text { def }}{=} \mathcal{D} q(\kappa) \\
& \quad \exp \left[-(-1)^{i} \mathcal{R}^{i}(q)(\kappa)\left(\epsilon^{\prime}(q(\kappa)) \overleftarrow{\partial}_{i}^{(q(\kappa))}\right)\right] \\
& \text { for } J(\kappa)=1-\left[(-1)^{i} \mathcal{R}^{i}(q)(\kappa)\left(\epsilon^{\prime}(q(\kappa)) \overleftarrow{\partial}_{i}^{(q(\kappa))}\right)\right]
\end{aligned}
$$

As we suppose that after a change of variables generated by FSUSY transformations, $q^{i} \rightarrow q^{i}(\kappa)$, the supersymmetric action $S(q)$ also changes to $S(q)+S_{1}(\kappa)$ with the local functional $S_{1}(q)$ vanishing at $\kappa=0$, the functional equation must hold:

$$
\begin{aligned}
& \int \mathcal{D} q(\kappa)\left[\frac{d}{d \kappa} \ln J(\kappa)-\frac{l}{\hbar} \frac{d}{d \kappa} S_{1}(q(\kappa))\right] \\
& \times \exp \left\{\frac{l}{\hbar}\left[S(q)+S_{1}(q(\kappa))\right]\right\} .
\end{aligned}
$$

The necessary condition that Eq. (24) be solvable is $S_{1}(q(\kappa))$ $\overleftarrow{s}=0$, i.e., the addition to the supersymmetric action must also be supersymmetric. Once again, FSUSY transformation with appropriate parameter $\epsilon$ change a supersymmetric action $S_{\text {susy }}$ to a new effective action $S_{\text {susy }}+S_{1}(\kappa=1)$ within the functional integration.

Note that one can perform a similar analysis in the case of an $N=1$ SUSY transformation with parameter $\bar{\epsilon}$ and the result would be the same. The only difference is that the parameter $\epsilon$ will be replaced everywhere by $\bar{\epsilon}$ and the generator $R_{1}^{i}(q)$ will be replaced by $R_{2}^{i}(q)$.

\section{Supersymmetric harmonic oscillator}

In this section, we analyze the $N=1$ supersymmetric free toy model with $(1,1)$ physical degrees of freedom described 
by one bosonic $x$ and two fermionic $\psi, \bar{\psi}$ coordinates: collectively, $q^{i}=(x, \psi, \bar{\psi}), i=1, \ldots, n ; n=(1,2)$ from the generalized SUSY perspectives. Here, we find that the (trivial) interaction terms for such a supersymmetric model emerges naturally through the Jacobian of functional measure. Let us start by writing the classical action $S(q)$ for the supersymmetric harmonic oscillator:

$S=\int_{t_{\text {in }}}^{t_{\text {out }}} \mathrm{d} t\left[\frac{1}{2} \dot{x}^{2}-\frac{1}{2} \omega^{2} x^{2}+i \bar{\psi} \dot{\psi}-\omega \bar{\psi} \psi\right]$.

This action refers to a free toy model with $(1,1)$ physical degrees of freedom, formally due to the presence of secondclass constraints for $\psi$. Here, we pass to dimensionless quantities, so that, for convenience, the mass $m=1$ for the bosonic part. The action is invariant up to a total time derivative with respect to an $N=1$ subalgebra of the total SUSY superalgebra with parameter $\epsilon^{1}=\epsilon(m=1)$,

$\delta_{\epsilon}[x, \psi, \bar{\psi}]=\frac{1}{\sqrt{2}}[\bar{\psi},-\imath \dot{x}-\omega x, 0] \epsilon \equiv[x, \psi, \bar{\psi}] \overleftarrow{s} \epsilon$,

and also with respect to an $N=1$ subalgebra with an odd parameter $\epsilon^{2}=\bar{\epsilon}$,

$\delta_{\bar{\epsilon}}[x, \psi, \bar{\psi}]=-\frac{1}{\sqrt{2}}[\psi \bar{\epsilon}, 0,-l \dot{x}+\omega x] \bar{\epsilon} \equiv[x, \psi, \bar{\psi}] \overleftarrow{s} \bar{\epsilon}$

which is related with the above subalgebra by means of complex conjugation, $\delta_{\bar{\epsilon}}[x, \psi, \bar{\psi}]=\left(\delta_{\epsilon}[x, \bar{\psi}, \psi]\right)^{*}$, determined by the rule

$$
\begin{aligned}
(x, \psi, \bar{\psi}, \epsilon, \bar{\epsilon})^{*} & =(x, \bar{\psi}, \psi, \bar{\epsilon}, \epsilon) \text { and }(a b)^{*} \\
& =b^{*} a^{*} \text { for } a, b \in\{x, \psi, \bar{\psi}, \epsilon, \bar{\epsilon}\} .
\end{aligned}
$$

$N=2$ SUSY algebraic transformations are determined by the identification $\epsilon^{a}=(\epsilon, \bar{\epsilon}), \psi^{a}=(\psi, \bar{\psi})$ :

$$
\begin{aligned}
\delta_{\epsilon_{a}}\left[x, \psi^{b}\right] & =\left[x, \psi^{b}\right] \overleftarrow{s}_{a} \epsilon^{a} \equiv R_{a}^{i}(q) \epsilon^{a}, \text { for } R_{a}^{i} \\
& =\frac{1}{\sqrt{2}}\left[\varepsilon_{c a} \psi^{c},\left[(-1)^{b} \iota \dot{x}-\omega x\right] \delta_{a}^{b}\right],
\end{aligned}
$$

whereas $N=2$ FSUSY transformations form an Abelian group $\left\{g\left(\epsilon^{a}\right)=\exp \left\{\overleftarrow{s}_{a} \epsilon^{a}\right\}\right\}$, and we have quadratic terms in powers of $(\epsilon)^{2}=\epsilon_{a} \epsilon^{a}=2 \epsilon \bar{\epsilon}$ together with finite transformations realized on $q^{i}$ are

$$
\begin{aligned}
{\left[x, \psi^{b}\right] \overleftarrow{s}^{a} \overleftarrow{s}_{a}(\epsilon)^{2} } & =\left[\omega x, \frac{1}{2}\left((-1)^{b} l-\omega\right) \psi^{b}\right](\epsilon)^{2} \\
\Longrightarrow \Delta_{\epsilon^{a}}\left[x, \psi^{b}\right] & =\delta_{\epsilon_{a}}\left[x, \psi^{b}\right]+\frac{1}{4}\left[x, \psi^{b}\right] \overleftarrow{s}^{a} \overleftarrow{s}_{a}(\epsilon)^{2}
\end{aligned}
$$

Then $S\left(\left[x, \psi^{b}\right] g\left(\epsilon^{a}\right)\right)=S\left(x, \psi^{b}\right)$ for arbitrary finite and FD $\epsilon^{a}$. SUSY invariant interaction terms (with respect to algebraic transformations) may (for $n>1$ ) be given by a polynomial in $x, \psi, \bar{\psi}$,

$$
\begin{aligned}
S_{\text {int }} & =\int_{t_{\text {in }}}^{t_{\text {out }}} \mathrm{d} t \sum_{n=2}^{M} g_{n}\left[-\frac{n}{\sqrt{2}} x^{n-1} \bar{\psi} \psi-\frac{i}{\sqrt{2}} x^{n} \dot{x}-\frac{1}{\sqrt{2}} \omega x^{n+1}\right] \\
& =\int_{t_{\text {in }}}^{t_{\text {out }}} \mathrm{d} t \sum_{n=2}^{M} g_{n}\left[\delta_{\epsilon}\left(x^{n} \psi\right)\right] \overleftarrow{\partial} \epsilon \\
& =-\int_{t_{\text {in }}}^{t_{\text {out }}} \mathrm{d} t \sum_{n=2}^{M} g_{n}\left[\frac{\sqrt{2}}{(n+1)} \delta_{\epsilon} \delta_{\bar{\epsilon}}\left(x^{n+1}\right)\right] \overleftarrow{\partial}_{\epsilon} \overleftarrow{\partial}_{\bar{\epsilon}} \\
& =-\int_{t_{\text {in }}}^{t_{\text {out }}} \mathrm{d} t \sum_{n=2}^{M} g_{n}\left[\frac{\sqrt{2}}{2(n+1)}\left(x^{n+1}\right) \overleftarrow{s}^{a} \overleftarrow{s}_{a}\right]
\end{aligned}
$$

with some coupling constants $g_{n}$ providing a dimension of the action. The interaction (31) appears trivial, due to definition (18). Then the full action incorporating the interaction, $S_{\text {full }}=S+S_{\text {int }}$, is invariant under the $N=1$ FSUSY transformations given in (26) and (27), as well as with respect to the $N=2$ FSUSY transformations (30).

The generators of the SUSY transformations (26), (27), and (29) can be represented through a standard SUSY representation with the help of the supercommutator [, \} for equal times:

$\delta_{\epsilon} q^{i}=i\left[q^{i}, Q\right\} \epsilon, \quad \delta_{\bar{\epsilon}} q^{i}=i\left[q^{i}, \bar{Q}\right\} \bar{\epsilon}$,

with an explicit realization of the supercharges

$$
\begin{aligned}
& (Q, \bar{Q})=\frac{1}{\sqrt{2}}\left(\overleftarrow{\partial}_{x} \bar{\psi}+\overleftarrow{\partial}_{\psi}[i \dot{x}+\omega x]\right. \\
& \left.\overleftarrow{\partial}_{x} \psi+\overleftarrow{\partial}_{\bar{\psi}}[-i \dot{x}+\omega x)\right]
\end{aligned}
$$

This is nothing else than $s$ and $\bar{s}$, respectively, satisfying the algebra (2).

\subsection{Generalized SUSY transformations and its Jacobians}

Following Sect. 2, we generalize the SUSY transformations (26), (27), and (30) by making the transformation parameters finite and field dependent:

$$
\begin{aligned}
\left(\delta_{\epsilon}, \delta_{\bar{\epsilon}}\right) q^{i} & =q^{i}(\overleftarrow{s} \epsilon(q), \overleftarrow{s} \bar{\epsilon}(q)) \text { and } \Delta_{\epsilon^{a}} q^{i} \\
& =q^{i}\left(\overleftarrow{s}_{a} \epsilon^{a}(q)+\frac{1}{4} \overleftarrow{s}^{a} \overleftarrow{s}_{a}(\epsilon(q))^{2}\right)
\end{aligned}
$$

Corresponding to $N=1$ and $N=2$ FSUSY transformations, the Jacobians of the change of variables in the path integral (8) question are given by (19): 


$$
\begin{aligned}
J(q(\epsilon)) & =(1+\epsilon \overleftarrow{s})^{-1} \text { and } J\left(q\left(\Lambda \overleftarrow{s}_{a}\right)\right) \\
& =\left(1+\frac{1}{2} \Lambda \overleftarrow{s}_{a} \overleftarrow{s}^{a}\right)^{-2}, \text { for } \epsilon_{a}(q)=\Lambda(q) \overleftarrow{s}_{a}
\end{aligned}
$$

where $\Lambda(q)$ is an arbitrary bosonic functional. When considering [31], the finite FD parameter $\epsilon(q)$ represented in terms of the infinitesimal $\epsilon^{\prime}(q(\kappa)$,

$\epsilon(q)=\int_{0}^{1} \mathrm{~d} \kappa \epsilon^{\prime}(q(\kappa))$ and $\bar{\epsilon}(q)=\int_{0}^{1} \mathrm{~d} \kappa \bar{\epsilon}^{\prime}(q(\kappa))$,

represents arbitrary finite FD SUSY parameters. The Jacobian for both $N=1$ SUSY transformations can be calculated using (22).

This shows that the interactions terms (31) may be generated by $N=1$ and $N=2$ FSUSY transformations with appropriate parameters.

\subsection{Generating the interaction terms}

To find an explicit finite FD parameter $\epsilon(q)$ for the $N=1$ SUSY transformation which generates the trivial interaction terms (31), we consider the functional equation

$Z_{0}=Z_{\text {int }}$ where $Z_{\text {int }}=\int \mathcal{D} q \exp \left\{\frac{l}{\hbar}\left[S(q)+S_{\text {int }}(q)\right]\right\}$,

and $Z_{0}$ is determined in (8). Making a change of variable in the integrand of $Z_{0}$ generated by FSUSY, we obtain the equation with an accuracy up to the total functional derivative:

$$
\begin{aligned}
i \hbar \ln J(q(\epsilon))+S_{\text {int }}(q) & =0 \Longleftrightarrow i \hbar \ln (1+\epsilon(q) \overleftarrow{s}) \\
& =\int_{t_{\text {in }}}^{t_{\text {out }}} \mathrm{d} t \sum_{n=2}^{M} g_{n}\left[x^{n} \psi\right] \overleftarrow{s}
\end{aligned}
$$

which we call a compensation equation. Because both parts of the compensation equation are $s$-exact, we determine the unknown $\epsilon(q)$ in terms of $S_{\text {int }}$,

$$
\begin{aligned}
\epsilon\left(q \mid S_{\text {int }}\right) & =\frac{l}{\hbar} g(y) \int_{t_{\text {in }}}^{t_{\text {out }}} \mathrm{d} t \sum_{n=2}^{M} g_{n}\left[x^{n} \psi\right], \text { for } g(y) \\
& =\frac{1-\exp \{y\}}{y}, y \equiv \frac{l}{\hbar} S_{\text {int }} .
\end{aligned}
$$

Vice-versa, considering Eq. (38) for some unknown interaction, we can always construct the trivial interaction, $S_{\text {int }}=$ $U(q) \overleftarrow{s}$ for any $N=1$ FSUSY transformation with given $\epsilon(q)$ :
$U(q \mid \epsilon)=\frac{\hbar}{l}\left[\sum_{n=1} \frac{(-1)^{n-1}}{n}(\epsilon \overleftarrow{S})^{n-1}\right] \epsilon$

The same can be done for an $N=1$ FSUSY with $\bar{\epsilon}(q)$ concerning a one-to-one correspondence among trivial interactions, represented as $\bar{U}(q) \stackrel{\overleftarrow{s}}{\varepsilon} \varepsilon(\bar{U}(q))=1$ and a set of respective $N=1$ FSUSY transformations.

Concerning the case of $N=2$ FSUSY transformations with $\epsilon^{a}(q), a=1,2$, the generation of trivial $N=2$ supersymmetric interactions is the same for functionally dependent $\epsilon^{a}(q)=\Lambda \overleftarrow{s}^{a}$. The corresponding compensation equation, providing (37) and its solution for the given interaction (31), with bosonic potential $U_{2}(q)=\sum_{n=2}^{M} g_{n} \frac{\sqrt{2}}{2(n+1)} x^{n+1}$ : $S_{\text {int }}=U_{2}(q) \overleftarrow{s} a \overleftarrow{s} a$, is

$$
\begin{aligned}
& i \hbar \ln J(q(\epsilon))+S_{\operatorname{int}}(q)=0 \Longleftrightarrow i \hbar \ln \left(1+\frac{1}{2} \Lambda(q) \overleftarrow{s}_{a} \overleftarrow{s}^{a}\right) \\
& =-U_{2}(q) \overleftarrow{s} a \overleftarrow{s}_{a} \\
& \Lambda\left(q \mid U_{2}\right) \overleftarrow{s}_{a}=\epsilon_{a}\left(q \mid U_{2}\right)=\frac{i}{2 \hbar} g(y) U_{2} \overleftarrow{s}_{a}, \Lambda\left(q \mid U_{2}\right) \\
& \quad=\frac{i}{2 \hbar} g(y) U_{2}, \text { for } y \equiv(i / 4 \hbar) U_{2} \overleftarrow{s}_{a} \overleftarrow{s}_{a}
\end{aligned}
$$

Conversely, for an unknown interaction we can always construct the trivial interaction, $S_{\mathrm{int}}=U_{2}(q) \overleftarrow{s} a \overleftarrow{s} a$, for any $N=2$ FSUSY transformation with a given $\epsilon^{a}(q)=\Lambda \overleftarrow{s} a$

$U_{2}\left(q \mid \epsilon_{a}\right)=4 \imath \hbar\left[\sum_{n=1} \frac{(-1)^{n-1}}{2^{n} n}\left(\Lambda \overleftarrow{s} a \overleftarrow{s}_{a}\right)^{n-1} \Lambda\right]$

Therefore, if the trivial interaction $S_{\mathrm{tr}}$ is given by $S_{\mathrm{tr}}=$ $U \overleftarrow{s}=\bar{U} \overleftarrow{s}=U_{2} \overleftarrow{s} a \overleftarrow{s} a$ then it can be generated (or removed from the initial action) by any $N=1,2$ FSUSY transformations with respective $\epsilon(q), \bar{\epsilon}(q), \epsilon^{a}(q)=\Lambda \overleftarrow{s} a$.

Omitting the details of a similar application of $N=1$ FSUSY transformations in the form (36) to derive the interaction (31), we stress that solving the problem amounts to calculating the Jacobian $J(\kappa)$ in Eq. (22). To find an unknown $S_{1}(q(\kappa), \kappa)$, we consider an infinitesimal FD parameter in the form

$\epsilon^{\prime}(q)=-\int_{t_{\text {in }}}^{t_{\mathrm{out}}} \mathrm{d} t \sum_{n=2}^{M}\left(g_{n} x^{n} \psi\right)$

We then choose the following ansatz for $S_{1}$ :

$$
\begin{aligned}
& S_{1}(q(\kappa), \kappa)=\int_{t_{\mathrm{in}}}^{t_{\mathrm{out}}} \mathrm{dt} \sum_{n=2}^{M} g_{n}\left(\chi_{1}(\kappa) x^{n-1} \bar{\psi} \psi+\chi_{2}(\kappa) x^{n} \dot{x}\right. \\
& \left.+\chi_{3}(\kappa) x^{n+1}\right),
\end{aligned}
$$

where $\chi_{i}, i=1,2,3$ are constant $\kappa$-dependent parameters satisfying the condition $\chi_{i}(\kappa=0)=0$. From Eq. (24), we derive the following differential equations (in $\kappa$ ): 
$\sqrt{2} \chi_{1}^{\prime}-n=0, \sqrt{2} \chi_{2}^{\prime}-\imath=0, \sqrt{2} \chi_{3}^{\prime}-\omega=0$,

whose obvious solution (when integrating from 0 to $\kappa$ )

$\left(\chi_{1}, \chi_{2}, \chi_{3}\right)=\left(\frac{n}{\sqrt{2}} \kappa, \frac{i}{\sqrt{2}} \kappa, \frac{1}{\sqrt{2}} \omega \kappa\right)$,

leads to an explicit form of $S_{1}(q(\kappa), \kappa)$, while $\kappa=1$ leads to $S_{\text {int }}(31)$.

\section{Conclusions}

We have extended the results and ideas of our previous study [31,62,66-69] considering the special abelian SUSY transformations as a symmetry of a Lagrangian action with bosonic and fermionic degrees of freedom, which form a superalgebra with $m$ Grassman-odd parameters. The SUSY invariance of the action for infinitesimal values of the parameters is restored to the case of finite values by solving the Lie equations. As the result, we have constructed, starting from a Lie superalgebra, a Lie supergroup [where the expcorrespondence completely maps the Lie superalgebra to the Lie supergroup (4)] with each its element having invariance transformation of the supersymmetric action in powers of the Grassman-odd parameters. This construction generalizes the case of BRST $(m=1)$ and BRST-antiBRST $(m=2)$ finite transformations for the gauge theories with a closed gauge algebra, including Yang-Mills theories. We have calculated the Jacobians of a change of variables in the path integral with a supersymmetric action, given by finite SUSY transformations with field-dependent parameters in (14), which contains as a partial case the Jacobians of formal BRST and BRST-antiBRST finite FD transformations. Because the set of FSUSY transformations satisfies the equivalence theorem conditions [74], the addition from the functional measure in the path integral may modify the supersymmetric action by a Jacobian more than quadratic powers of fields that still leads to the same conventional $S$-matrix. We have called such additions to the action trivial interactions. Non-trivial FSUSY invariant interactions cannot be generated by this receipt. It is shown that the presence of $m$-parametric FSUSY transformations leads to the presence of standard Ward identities for generating functionals of Green functions (20) corresponding to the constant odd parameters, as well as to a modified Ward identity (21) depending on FD finite odd parameters $\epsilon^{\alpha}(q)$.

We have illustrated these results by a simple free toy model with $(1,1)$ physical degrees of freedom describing a supersymmetric harmonic oscillator by a generalization of $N=1$ and $N=2$ SUSY transformations. It is shown that any trivial interactions can be completely generated from the functional measure by means of $N=1$ and $N=2$ FSUSY transformations, respectively, with FD parameter and functionallydependent parameters.
The present research may be used to analyze the influence on the structure of a quantum action of real space-time SUSY transformations with FD parameters, which, however, do not form an Abelian superalgebra and contain, in addition to $Q$ and $\bar{Q}$, also a Grassman-even generator of momenta, $P^{\mu}$. At the same time, in the case of the additional presence of the gauge invariance for a supersymmetric action the problem of a joint consideration of FSUSY transformations and real BRST or BRST-antiBRST symmetry transformations for a quantum action may prove to be a promising direction of research.

Acknowledgments The authors are grateful to P.Yu. Moshin for interest and discussions. The study of A.R. was supported by the RFBR grant No. 16-42-700702.

Open Access This article is distributed under the terms of the Creative Commons Attribution 4.0 International License (http://creativecomm ons.org/licenses/by/4.0/), which permits unrestricted use, distribution, and reproduction in any medium, provided you give appropriate credit to the original author(s) and the source, provide a link to the Creative Commons license, and indicate if changes were made. Funded by SCOAP ${ }^{3}$.

\section{References}

1. M. Green, J. Schwarz, E. Witten, Superstring Theory (Cambridge University Press, Cambridge, 1987)

2. S. Ferrara, D. Freedman, P. van Nieuwenhuizen, Phys. Rev. D 13, 3214 (1976)

3. S. Deser, B. Zumino, Phys. Lett. B 62, 335 (1976)

4. I.L. Buchbinder, E.A. Ivanov, I.B. Samsonov. arXiv:1603.02768 [hep-th]

5. E.A. Ivanov. arXiv: 1604.01379 [hep-th]

6. M. Vasiliev, Lect. Notes Phys. 892, 227 (2015)

7. A. Fotopoulos, M. Tsulaia, Int. J. Mod. Phys. A. 24, 1 (2008). arXiv:0805.1346 [hep-th]

8. I.L. Buchbinder, V.A. Krykhtin, A.A. Reshetnyak, Nucl. Phys. B. 787, 211 (2007)

9. A.A. Reshetnyak, Nucl. Phys. B. 869, 523 (2013)

10. R.R. Metsaev, Phys. Lett. B. 720, 237 (2013)

11. I.L. Buchbinder, T.V. Snegirev, YuM Zinoviev, JHEP 1510, 148 (2015)

12. F. Cooper, A. Khare, U.P. Sukhatme, Supersymmetry in Quantum Mechanics (World Scientific, Singapore, 2001)

13. E. Witten, Nucl. Phys. B 188, 513 (1981)

14. F. Cooper, B. Freedman, Ann. Phys. 146, 262 (1983)

15. P. Salomonson, J. van Holten, Nucl. Phys. B 196, 509 (1982)

16. M. Bernstein, L. Brown, Phys. Rev. Lett. 52, 1933 (1984)

17. P. Kumar, M. Ruiz-Altaba, B.S. Thomas, Phys. Rev. Lett. 57, 2749 (1986)

18. F. Marchesoni, P. Sodano, M. Zannetti, Phys. Rev. Lett. 61, 1143 (1988)

19. W.-Y. Keung, E. Kovacs, U. Sukhatme, Phys. Rev. Lett. 60, 41 (1988)

20. C. Becchi, A. Rouet, R. Stora, Phys. Lett. B 52, 344 (1974)

21. C. Becchi, A. Rouet, R. Stora, Ann. Phys. 98, 287 (1976)

22. I.V. Tyutin, Lebedev Inst. 39 (1975). arXiv:0812.0580 [hep-th] (preprint)

23. C. Rupp, R. Scharf, K. Sibold, Nucl. Phys. B 612, 313 (2001)

24. P. Van Nieuwenhuizen. arXiv:hep-th/0408179 
25. B. de Witt, J.W. van Holten, Phys. Lett. B 79, 389 (1979)

26. I.A. Batalin, G.A. Vilkovisky, Phys. Lett. B 102, 27 (1981)

27. E.S. Fradkin, G.A. Vilkovisky, Phys. Lett. B 55, 224 (1975)

28. I.A. Batalin, G.A. Vilkovisky, Phys. Lett. B 69, 309 (1977)

29. M. Henneaux, C. Teitelboim, Quantization of Gauge Systems (Univ. Press, Princeton, 1992)

30. S. Weinberg, The Quantum Theory of Fields, Vol-II: Modern Applications (Univ Press, Cambridge, 1996)

31. S.D. Joglekar, B.P. Mandal, Phys. Rev. D 51, 1919 (1995)

32. S. Upadhyay, Phys. Lett. B 723, 470 (2013)

33. S. Upadhyay, Ann. Phys. 344, 290 (2014)

34. S.D. Joglekar, B.P. Mandal, Int. J. Mod. Phys. A 17, 1279 (2002)

35. R. Banerjee, B.P. Mandal, Phys. Lett. B 488, 27 (2000)

36. S. Upadhyay, S.K. Rai, B.P. Mandal, J. Math. Phys. 52, 022301 (2011)

37. S. Upadhyay, B.P. Mandal, Phys. Lett. B 744, 231 (2015)

38. S. Upadhyay, B.P. Mandal. arXiv:1407.2017 [hep-th]

39. S. Upadhyay, B.P. Mandal, Prog. Theor. Exp. Phys. 053B04 (2014)

40. S. Upadhyay, B.P. Mandal, Eur. Phys. J. C 72, 2065 (2012)

41. S. Upadhyay, B.P. Mandal, Ann. Phys. 327, 2885 (2012)

42. S. Upadhyay, B.P. Mandal, EPL 93, 31001 (2011)

43. S. Upadhyay, B.P. Mandal, Mod. Phys. Lett. A 25, 3347 (2010)

44. B.P. Mandal, S.K. Rai, S. Upadhyay, EPL 92, 21001 (2010)

45. S. Upadhyay, M.K. Dwivedi, B.P. Mandal, Int. J. Mod. Phys. A 28, 1350033 (2013)

46. M. Faizal, B.P. Mandal, S. Upadhyay, Phys. Lett. B 721, 159 (2013)

47. R. Banerjee, B. Paul, S. Upadhyay, Phys. Rev. D 88, 065019 (2013)

48. S. Upadhyay, Ann. Phys. 356, 299 (2015)

49. S. Upadhyay, Phys. Lett. B 740, 341 (2015)
50. S. Upadhyay, EPL 105, 21001 (2014)

51. S. Upadhyay, Phys. Lett. B 727, 293 (2013)

52. S. Upadhyay, EPL 104, 61001 (2013)

53. S. Upadhyay. arXiv:1308.0982 [hep-th]

54. R. Banerjee, B. Paul, S. Upadhyay, Phys. Rev. D 88, 065019 (2013)

55. S. Upadhyay, D. Das, Phys. Lett. B 733, 63 (2014)

56. R. Banerjee, S. Upadhyay, Phys. Lett. B 734, 369 (2014)

57. S. Upadhyay, Ann. Phys. 340, 110 (2014)

58. P. Lavrov, O. Lechtenfeld, A. Reshetnyak, JHEP 1110, 043 (2011)

59. V.N. Gribov, Nucl. Phys. B. 139, 1 (1978)

60. D. Zwanziger, Nucl. Phys. B. 323, 513 (1989)

61. P. Lavrov, O. Lechtenfeld, Phys. Lett. B. 725, 382 (2013)

62. A. Reshetnyak, Int. J. Mod. Phys. A 29, 1450128 (2014)

63. G. Curci, R. Ferrari, Phys. Lett. B. 63, 91 (1976)

64. L. Alvarez-Gaume, L. Baulieu, Nucl. Phys. B. 212, 255 (1983)

65. V.P. Spiridonov, Nucl. Phys. B. 308, 527 (1988)

66. P.Y. Moshin, A.A. Reshetnyak, Nucl. Phys. B. 888, 92 (2014)

67. P.Y. Moshin, A.A. Reshetnyak, Phys. Lett. B 739, 110 (2014)

68. P.Y. Moshin, A.A. Reshetnyak, Int. J. Mod. Phys. A 29, 1450159 (2014)

69. P.Y. Moshin, A.A. Reshetnyak, Int. J. Mod. Phys. A 30, 1550021 (2014)

70. P.Y. Moshin, A.A. Reshetnyak. arXiv:1506.04660 [hep-th]

71. P.Y. Moshin, A.A. Reshetnyak. arXiv:1604.03027 [hep-th]

72. L.D. Faddeev, V.N. Popov, Phys. Lett. B. 25, 29 (1967)

73. I.A. Batalin, P.M. Lavrov, I.V. Tyutin, Int. J. Mod. Phys. A. 29, 1450166 (2014)

74. K.E. Kallosh, I.V. Tyutin, Sov. J. Nucl. Phys. 17, 98 (1973) 